\title{
A Qos Based Mac Protocol for Wireless Multimedia Sensor Network
}

\author{
Smita Pawar ${ }^{1}$, Prabha Kasliwal ${ }^{2}$ \\ ${ }^{1}$ Lecturer, E\&TC Department, Maharashtra Academy of Engineering, Alandi1, Pune University, India \\ ${ }^{2}$ Assosiate Professor, Electronics Department, Maharashtra Academy of Engineering, Alandi2, Pune University, \\ India
}

\begin{abstract}
Wireless Multimedia Sensor Networks (WMSNs) have emerged and shifted the focus from the typical scalar wireless sensor networks to networks with multimedia devices that are capable to retrieve video, audio, images, as well as scalar sensor data. WMSNs are able to deliver multimedia content due to the availability of inexpensive CMOS cameras and microphones coupled with the significant progress in distributed signal processing and multimedia source coding techniques. MAC in WMSNs is essential to coordinate the channel access among competing devices. Therefore, a proposal of MAC layer protocol for WMSNs should satisfy the following feature like Maximize network throughput, Enhance transmission reliability, and Minimize control overhead, be energy-efficient, Guarantee a certain level of QoS In this project, using NS2 simulation tool, we have proposed a new QoS-based sensory MAC protocol, which not only adapts to application oriented QoS, but also attempts to conserve energy without violating QoS-constraints.
\end{abstract}

Key words: MAC protocol, QoS, Multimedia sensor networks, CSMA/CA, Duty cycle, NS2 Simulator.

\section{Introduction}

Wireless communication is a gradually changing paradigm from its existing voice-alone services to a new world of real-time audio-visual applications. This ever-increasing popularity of multimedia applications has already started penetrating the domain of wireless sensor networks - thereby giving birth to the new terminology wireless multimedia sensor networks (WMSNs)[2] .Video surveillance, telemedicine and trafficcontrol are going to be the killer-applications of this emerging WMSN must overcome many constraints. While the need to minimize the energy consumption has driven significant researches in wireless sensor networks, offering some precise quality of service (QoS) [1] for multimedia transmission over sensor networks has not received significant attention. In this project, a new QoS-based sensory MAC protocol is proposed, which not only adapts to application oriented QoS, but also attempts to conserve energy without violating QoS-constraints. This motivates to look for QoS-based, yet energy-aware, MAC protocols for WMSNs. The objective of this work is to develop a new QoS-based, energy- aware MAC protocol for WMSNs. More specifically contributions are:

(1) To develop a QoS-based MAC protocol for WMSNs with dynamic adaptation of contention window-sizes

(2)The protocol is also designed to dynamically adjust its duty cycle based on the major application traffic.

\subsection{Requirements of MAC Protocol for WMSN}

MAC in WMSNs is essential to coordinate the channel access among competing devices. Given the energy constraints of the small, battery powered sensor devices, it is desirable that the MAC layer provides reliable, error-free data transfer with minimum efficient resource utilization. MAC layer attempts to address these issues by enforcing channel access, scheduling policies and error control, minimize control overhead, Be energy-efficient, guarantee of QoS.

\subsection{Existing MAC Protocol}

In order to decrease or if possible to eliminate various sources of energy wastage, several protocols has been proposed which are divided into two main classes

(1)TDMA-based protocols: these protocols known as deterministic are employed to avoid collisions by associating a slot time for each sensor node in a given cluster, and to mitigate the effects of overhearing problem, because in this situation each node knows his corresponding slot time to transmit data packet [3]. However, these protocols require the presence of a management authority (for example a dedicated access point) to orchestrate the various activities inside a cluster. This makes the use of these protocols more complex in the WSN where the nodes in general have a same priority and very limited resources.

(2)Contention-based protocols: these protocols known as CSMA-based are usually used in the multi-hop wireless networking due to their simplicity and their adequacy to be implemented in a decentralized environment like WSN. When these protocols are used, collisions can be occur in case of a receiver is located in the radio range of at least two sensor nodes transmitting simultaneously data packets to it. In this situation, this node will not succeed in receiving any data packet. These collisions generate useless retransmissions 
which cause energy consumption wastage and time consuming in data transmission. To decrease these collisions and to reduce considerably other sources of energy wastage, the Wake-up/Sleep mechanisms and/or the control messages RTS/CTS/ACK defined in 802.11x standard [8], are used to design energy efficient MAC protocols for WSN like S-MAC, T-MAC, B-MAC and Z-MAC. In our paper we compare our protocol with SMAC [8] and TMAC [5].

A node's radio, which by large consumes the majority of the energy, can be in one of four modes: transmit, receive idle listening (in which the radio is on but idle) and sleep. Energy cost in idle listening is almost identical to that of the receive mode, while the consumption in sleep mode is significantly lower than that of receiving. It is also known that the largest contribution to energy waste in MAC protocols is uneventful idle listening. Since a node has no explicit knowledge of when packets are sent for it from one of its neighbors, it must consistently keep its radio in listening node. To address this challenge and reduce the energy waste due to idle listening, several MAC protocols suited for sensor networks have emerged, including S- MAC [8] and TMAC [5]. These protocols incorporate some form of duty-cycle management that periodically sets each of the nodes in sleep mode so as to minimize the power consumption. However, in most protocols each node determines the duty cycle as a function of its own traffic load, thereby inherently limiting the overall performance of the network. In this paper we propose an optimization framework that generally captures several parameters pertaining to the dynamics of the MAC layer, and develop a practical algorithm based on dynamic duty cycle that makes the contention window adaptive in natural MAC protocol policy.

The proposed optimization scheme is simple, inherently distributed and self-organized. Although provisioning QoS in MAC layers for wireless cellular and local area networks is an active research area, QoSbased MAC protocol for wireless sensor networks have received relatively less attention.[1]

\subsection{Flowchart of Basic CSMA CA technique}

\section{Algorithms And Flowchart}

CSMA methods generally offer a lower delay and better throughput, especially at lower traffic loads; we also explore the CSMA methods to develop our newly proposed QoS- based MAC protocol for WMSNs

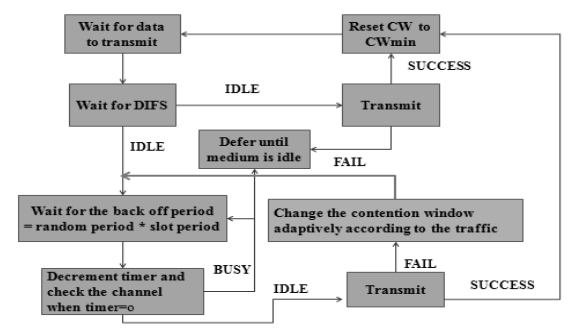

Fig. 1 CSMA/CA technique

\subsection{Algorithm for QoS-based energy efficient MAC protocol for WMSNs}

(1) Initially enough data is collected for statistics. After every d seconds the algorithm checks the number of packets transmitted and proceeds to next step only if more than Q packets are transmitted.

(2) In the next step, the instantaneous probability of transmission failure (Prf (t)) at any time instance $t$ is estimated using the number of transmission failures and number of transmission successes

(3) If packet failure at time $t$ is greater than packet failure at time $t-1$ then gradually increase the $\mathrm{CW}$ according to the equation (1) and (2) given below

(4) If packet failure at time t is greater than packet failure at time t-1 then do not change the CW

It should be noted that this CWtarget is not the size that each sensor node will always tune its CW to be, rather it is used to guide the direction of $\mathrm{CW}$ adjustment. The actual step size is a determined by both the CWtarget and the current contention window (CWcur), according to the following equation:

$\Delta \mathrm{CW}=(\mathrm{CW}$ target $-\mathrm{CWcur}) / \mathrm{Cwcur}$

where $\alpha$ is a QoS-based scaling factor.

(1) $\mathrm{CWcur}=\mathrm{CWcur}+\Delta \mathrm{CW}$

\subsection{Algorithm for Traffic Classification}

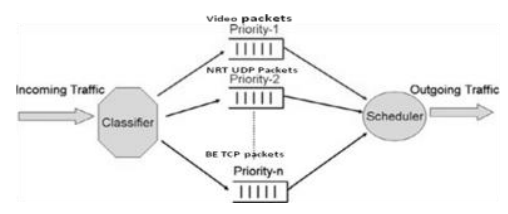

Fig .2 Traffic classifications 


\section{A Qos Based Mac Protocol For Wireless Multimedia Sensor Network}

(1) After every T seconds the algorithm checks number of packets transmitted and proceeds to next step only if more than $\mathrm{Q}$ packets are transmitted.

(2) In the next step it classifies the traffic into different categories based on the traffic class or type of service (ToS).For example, streaming multimedia, non-real-time (NRT) and best effort (BE) traffic are categorized as class- 1 , class- 2 and class- 3 , respectively.

(3) Now the algorithm determines the traffic class or ToS which dominated the traffic in the previous time frame T. In other words it finds the traffic class to which most packets belong to.

(4) Depending on this dominating traffic class the algorithm selects the active time (TA) and adjusts the duty cycle. Maintaining a synergy with the traffic priorities, the active time for streaming real-time, non-realtime (NRT) and best effort traffic is taken according to the degree of service differentiation these three different traffic types have in a relative sense.

(5) The sensory node keeps listening and potentially transmitting during its active period. In absence of any event during the entire activation period, the sensory node goes into the idle state. The strategy moves all packet transmission and reception into variable-size bursts. Thus, essentially the duty cycle of our proposed MAC protocol dynamically changes with the current traffic condition.

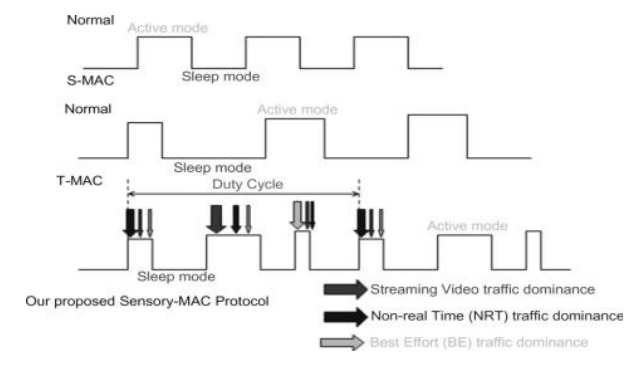

Fig .3 Dynamics of duty cycle in SMAC, TMAC and proposed MAC

As shown in Fig.3 below while S-MAC and T-MAC respectively operate on a static and thresholdbased duty cycle, our proposed MAC protocol adjusts it depending on the dominating traffic received. The active period is highest for delay-sensitive streaming multimedia traffic and lowest for BE traffic. With an adaptive duty cycle, we are thereby making the sleep and listen time dependent on traffic instead of having fixed as in S-MAC and T-MAC. Since the sensor On-time varies with the traffic we can compare total packet read, sent and received for different traffic, which can help us to calculate important performance parameters like Throughput, End - End delay, Packet delivery ratio etc

\section{Experimental Framework}

We will develop our sensor networks simulator based on NS2

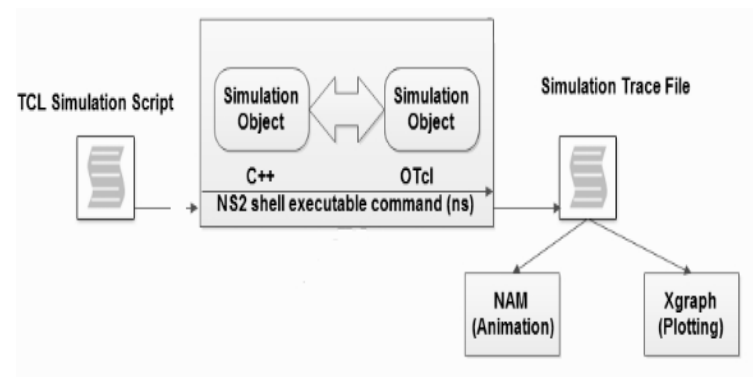

Fig. 4 NS2 structure

Network Simulator (Version 2), widely known as NS2, is simply an event driven simulation tool that has proved useful in studying the dynamic nature of communication networks. NS2 consists of two key languages: C++ and object-oriented Tool Command Language (OTcl) NS2 is the s/w package with the basic components like TCL/T k, OTcl, TCLCL as shown.TCL is open script language while Tk is the development tool for graphical interface OCTL object oriented TCL while TCLCL provides interface of Ns2 and OTcl .NS2 provides selected Xgraph, GNU plots and selectable component NAM. The traffic classification, priority assignment, priority- based back-off, sleeping and idle listening is simulated as different states in the simulator. 


\section{Methodology}

- Create a WSN topology in NS2 environment

- Accept the traffic after every $\mathrm{Q}$ sec

- Classify the traffic into RT, NRT and BE

- Adjust the contention window as per scaling factor and 802.11e EDCA parameter set.

- $\quad$ Run AWK and PERL file for End-to-End delay and Throughput

- Compare the results with the other MAC protocol

For our simulation, the scaling factor $\alpha$, used to determine the traffic priorities, is taken to be in proportion to the IP-ToS. Hence for our simulation, the $\alpha$ values are taken such

that they adjust the contention window between Cwmin and CWmax. Since the proposed protocol is QOS based we have taken 802.11e EDCA parameter set [3] for our reference.

Table 1. Different Parameter Settings in Ns2

\begin{tabular}{|l|l|}
\hline Number of nodes & 300 \\
\hline Channel capacity & 1Mbps \\
\hline$\alpha$ & 15 for Streaming Video \\
& 28.28 for Non Real Time \\
& 40 for Best Effort \\
\hline CWmax & 31 for Streaming Video \\
& 64 for Non Real Time \\
& 128 for Best Effort \\
\hline CWmin & 15 for Streaming Video \\
& 31 for Non Real Time \\
& 64 for Best Effort \\
\hline
\end{tabular}

\section{RESULTS}

Fig.5 explains the throughput-dynamics for different traffic classes. In our proposed MAC protocol we first classify the traffic into different classes depending on the type of service (ToS). Subsequently, it adjust it's the contention windows depending on the traffic classes and the QoS-demands for every traffic class. The streaming video traffic is given the highest priority, thereby achieving the lowest contention window $(1 / 4$ th of the BE traffic). The NRT traffic is given the second priority with contention window $1 / 2$ of the BE's contention window. Thus, as shown in Fig. 6, the streaming video traffic achieves a throughput of $50 \mathrm{Kbps}$ followed by the $30 \mathrm{Kbps}$ throughput of NRT traffic. The BE traffic attains a lowest throughput of $10 \mathrm{Kbps}$.

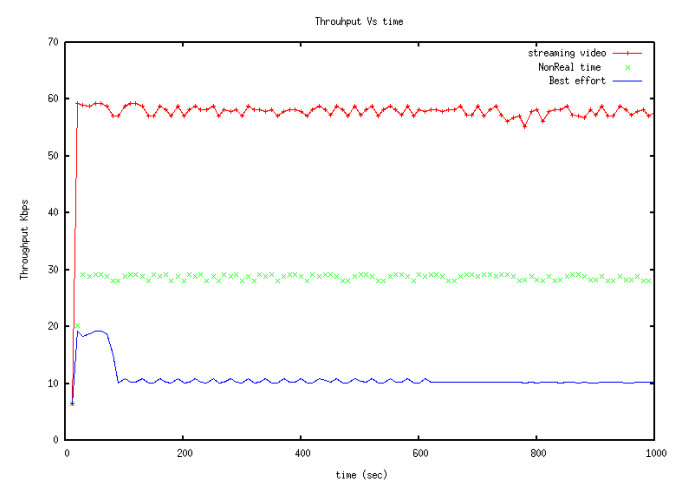

Fig.5 MAC throughput

The delay offered, for different traffic classes, by our proposed MAC protocol is shown in Fig. 6. The lower contention window of streaming video traffic gives it a delay of approx10 ms. The corresponding delay associated with NRT traffic is $50 \mathrm{~ms}$. The BE traffic attains highest average delay of $100 \mathrm{~ms}$ The traffic differentiation and subsequent adjustment of contention window (depending on traffic class) helps in the reduction of the delay for streaming multimedia traffic to pretty low values. This aids in successful multimedia transmission over wireless sensor networks with improved QoS. 


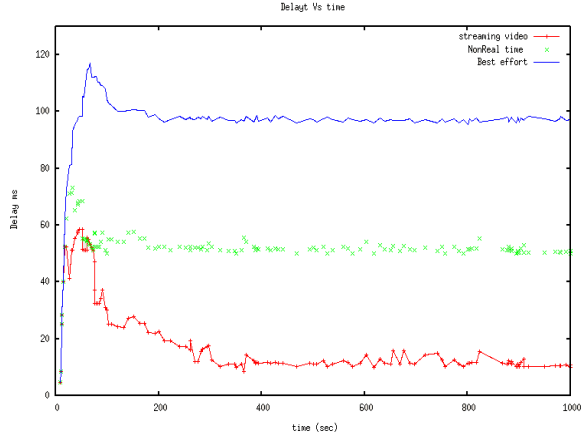

Fig. 7 depicts a comparative study of the wireless throughput between our protocol, SMAC and TMAC. It is clear that while SMAC and TMAC respectively achieve a throughput of $20 \mathrm{Kbps}$ and $10 \mathrm{Kbps}$, our protocol, achieves an average throughput of 50 Kbps. Fig 8 depicts impact of the node density on throughput. Throughput is defined as the maximum rate at which each node can send data to its destination in the network with the node density [6]. As number of nodes increases the hop progress increases while the hop count reduces. However large no of nodes may lead to contention thus degrading the throughput. Delay refers to end to end packet transmission delay in a network with node density n. Delay is directly proportional to hop count.

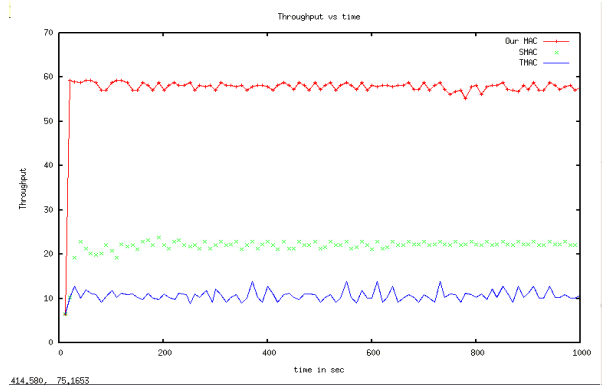

Fig.7 Comparative throughput of SMAC, TMAC and proposed protocol

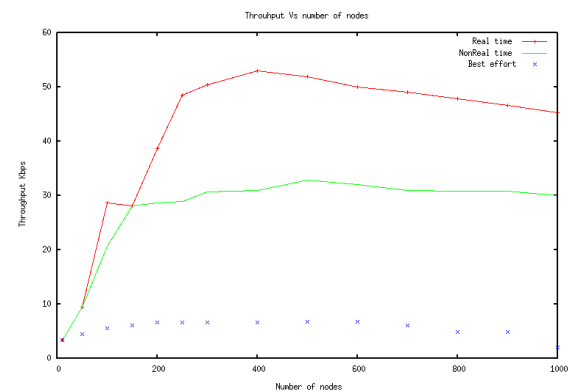

Fig. 8 Impact of node density on throughput

As shown in fig. 8 throughput degrades gradually after numbers of nodes are greater than 600.Thus as the number of nodes increases contention starts increasing which degrades the throughput. When the number increases to 1000 , the throughput decreases by 5 percent

Fig.9 Impact of node density on delay

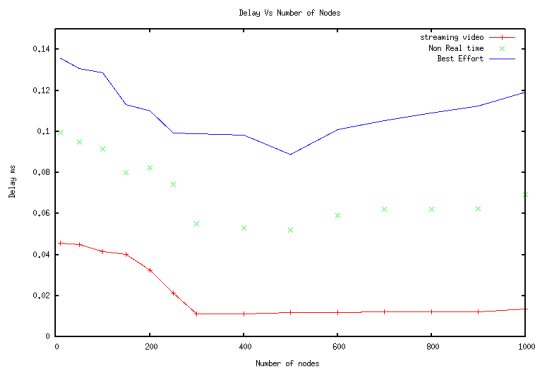




\section{A Qos Based Mac Protocol For Wireless Multimedia Sensor Network}

Fig 9 depicts impact of the node density on delay [6]. The average delay is obtained by averaging the delay experienced by each packet overall Source to Destination pairs, and this value is proportional to the expected hop count for each Source-Destination pair in the network.

\section{CONCLUSION}

To solve the problem of throughput and end to end delay in SMAC and TMAC we have proposed a MAC protocol with adaptive contention window. Our protocol can offers very high throughput and low delay characteristics, even in the presence of high traffic load. The scaling factor which makes the $\mathrm{CW}$ adaptive in nature is selected as per the 802.11e standard taking QoS into consideration. This paper also shows the impact of node density on the achievable throughput and the end-to-end delay for each Source-Destination pair in multi-hop wireless networks

\section{REFERENCES}

[1] Akkaya K., Younis M," An energy-aware QoS routing protocol for wireless sensor networks", in: Proceedings of the 23rd InternationalConference on Distributed Computing Systems (ICDCS), 2003.

[2] Akyildiz I.F., Melodia T.,Chowdhury K.R, "A survey on wireless multimedia sensor networks", The International Journal of Computer and Telecommunications Networking 51(4) (2007) 921-960

[3] Bianchi G, "Performance analysis of the IEEE 802.11 distributed Coordination function", IEEE Journal of Selected Areas of Communications 18 (3) (2000).

[4] Chen D, Varshney P.K., "QoS support in wireless sensor networks: A survey", in: Proceedings of International Conference on Wireless Networks (ICWN), 2004

[5] Dam T. V. and Langendoen K., "An adaptive energy-efficient mac protocol for wireless sensor networks," in SenSys '03: Proceedings of the 1stinternational conference on Embedded networked sensor systems, (NewYork, NY, USA), pp. 171-180, ACM Press, 2003

[6 Kuo Jia-Chun, Liao Wanjiun, and Hou Ting-Chao," Impact of Node Density on Throughput and Delay Scaling in Multi-Hop Wireless Networks", IEEE Transaction wireless communications, VOL. 8, NO. 10, October 2009

[7] Saxena Navrati , Roy Abhishek, Shin Jitae,"Dynamic duty cycle and adaptive contention window based QoS-MAC protocol for wireless multimedia sensor networks",Computer Network 52 (2008) 2532-2542

[8] Ye W, Heidemann J, and. Estrin D, "Medium access control with coordinated adaptive sleeping for wireless sensor networks," IEEE/ACMTrans. on Networks, vol.12, no. 3, pp. 493-506, 2004. 